\title{
CENTRALIZED EFFECT ON EXPECTED COSTS IN A MULTI-LOCATION NEWSBOY PROBLEM
}

\author{
Pao-Long Chang \\ National Chiao-Tung University
}

\author{
Chin-Tsai Lin \\ National Chiao-Tung University \\ and \\ Tamkang University
}

(Received May 8, 1989; Final August 27, 1990)

\begin{abstract}
This is a single-period single-product inventory model with several individual sources of demand. It is a multi-location problem with an opportunity for centralization. The holding and penalty cost functions, at each location, are assumed to be linear and identical. Two types of inventory system are considered in this paper: The decentralized system and the centralized system. The decentralized system is a system in which a separate inventory is kept to satisfy the demand at each source of demand and there is no reinforcement between locations; i.e., the surplus supplied location is not allowed to supply the deficient supplied location. Therefore, the total expected cost of the decentralized system is the sum of the expected cost of individual locations, as the holding cost or penalty cost of each location depends only on its own inventory level and is unrelated to any other location's inventory level. The centralized system is a system in which the surplus location is allowed to supplement the deficient one by transportation. Therefore, the holding cost or penalty cost is calculated by the net surplus or net deficient after the reinforcement. The total expected cost of the centralized system should therefore include the expected holding cost, the expected penalty cost and the expected transportation cost.

Let $\bar{h}>0, \bar{p}>0$ and $\bar{t} \geq 0$ be the unit holding cost, the unit penalty cost and the unit transportation cost, respectively. This paper demonstrates that, for any probability distribution of a location's demand, the following two properties are always true:
\end{abstract}

(1) $\bar{t}<\bar{h}+\bar{p}$ if and only if the total expected cost in a decentrahized system exceeds those in a centralized system.

(2) For all $i, j, i \neq j, p_{i j}$, the coefficient of correlation between the $i$-th location's demand and the $j$-th location's demand, is equal to 1 , then for any $\bar{t}$, the total expected cost in a decentralized system is equal to those in a centralized system.

\section{Introduction}

Eppen [1] presented a multi-location newsboy problem with normal distributions of a location's demand, and identical linear holding and penalty cost functions. The consolidation of demands from several facilities is considered, and his model demonstrates that:

(i) The expected holding and penalty costs in a decentralized system exceeds those in a centralized system;

(ii) For all $i, j, i \neq j, p_{i j}$, the coefficient of correlation between the $i$-th location's demand and the $j$-th location's demand, is equal to 1 , then the expected holding and penalty costs in a decentralized system are equal to those in a centralized system.

Stulman [3] considers the distribution of a location's demand as Poisson, and indicates that when the location's demand can be approximated by a normal distribution, then a centralized inventory is less costly than a non-centralized one. His method parallels the one 
treated by Eppen.

Although Eppen [1] gave a concrete discussion on the effects of centralization of this multi-location newsboy problem, the following two factors are still ignored in his model:

(a) The total expected cost of the centralized system includes only the holding costs and the penalty costs without taking into consideration the transportation costs from the surplus supplied locations to the deficient supplied locations.

(b) The probability distribution of a location's demand is assumed to be normal distribution. However, a location's demand is nonnegative whereas the normal distribution possesses positive probabilities to negative numbers. Therefore the following assumption is required in Eppen's model: the coefficient of variation of a location's demand is sufficiently small for the probabilities of negative demands to be neglected.

In this paper, we adopt a quite different method to show that:

(1) $\bar{t}<\bar{h}+\bar{p}$ if and only if the total expected cost in a decentralized system exceeds those in a centralized system.

(2) For all $i, j, i \neq j, p_{i j}$, the coefficient of correlation between the $i$-th location's demand and the $j$-th location's demand, is equal to 1 , then for any $\bar{t}$, the total expected cost in a decentralized system is equal to those in a centralized system. If $\bar{t}=0$, then the centralized system we considered in this paper, is identical to Eppen's centralized system. Hence properties (1) and (2) are generalizations of Eppen's results (i) and (ii), respectively.

\section{Assumptions and notations}

We adopt the following assumptions and notations:

$X_{i}=$ the demand at the $i$-th location with the probability density distribution $f_{i}(x)$, where $f_{i}(x)=0$ for all $x<0$ and $f_{i}(x)>0$, for all $x \geq 0$.

$p_{i j}=$ the correlation coefficient of $X_{i}$ and $X_{j}$.

$X_{0}=X_{1}+X_{2}+\cdots+X_{n}$; the total demand in the whole system with the probability density distribution $f_{0}\left(x_{0}\right)$, where $f_{0}\left(x_{0}\right)=0$, for all $x_{0}<0$.

$s_{i}=$ the stock level at the $i$-th location.

$s_{0}=s_{1}+s_{2}+\cdots+s_{n}$; the total stock level in the whole system.

$f(x)=$ the joint probability density distribution function of the demands $X=\left(X_{1}, X_{2}, \cdots, X_{n}\right)$.

$t(x)=$ the transportation cost function with the properties $t(x)=0$, for all $x \leq 0$ and $t(x)=\bar{t} \cdot x$, for all $x>0$.

$h(x)=$ the holding cost function with the properties $h(x)=0$, for all $x \leq 0$ and $h(x)=$ $\bar{h} \cdot x$, for all $x>0$.

$p(x)=$ the penalty cost function with the properties $p(x)=0$, for all $x \leq 0$ and $p(x)=\bar{p} \cdot x$, for all $x>0$.

$[y]^{+}=\max \{y, 0\}$ for any real number $y$. By using this, $t(x), h(x)$, and $p(x)$ can be expressed as, respectively, $\bar{t} \cdot[x]^{+}, \bar{h} \cdot[x]^{+}$, and $\bar{p} \cdot[x]^{+}$.

\section{The model of inventory systems}

\subsection{The decentralized inventory system}

The decentralized system is a system in which a separate inventory is kept to satisfy the demand at each source of demand and there is no reinforcement between location's demand. Its aim is to minimize the expected total cost $H_{D}(s)$, which is the sum of the expected 
locations costs $H_{i}\left(s_{i}\right)$; that is, to minimize separately the following $n$ functions.

$$
H_{i}\left(s_{i}\right)=\int_{0}^{\infty}\left(\bar{h} \cdot\left[s_{i}-x_{i}\right]^{+}+\bar{p} \cdot\left[x_{i}-s_{i}\right]^{+}\right) f_{i}\left(x_{i}\right) d x_{i}
$$

That is,

$$
\begin{aligned}
\min _{s} H_{D}(s) & =\sum_{i=1}^{n} \min _{s_{i}} H_{i}\left(s_{i}\right) \\
& =\int_{0}^{\infty} \cdots \int_{0}^{\infty}\left[\sum_{i=1}^{n} \bar{h} \cdot\left[s_{i}-x_{i}\right]^{+}+\sum_{i=1}^{n} \bar{p} \cdot\left[x_{i}-s_{i}\right]^{+}\right] f(x) d x
\end{aligned}
$$

where $s=\left(s_{1}, s_{2}, \cdots, s_{n}\right)$ and $x=\left(x_{1}, x_{2}, \cdots, x_{n}\right)$ are vectors.

\subsection{The centralized inventory system}

The centralized system is a system in which the surplus supplied location is allowed to supply the deficient supplied location by transportation and the holding cost or penalty cost is calculated by the net surplus or net deficient after the reinforcement. The total expected cost of the centralized system should therefore include the expected holding cost, the expected penalty cost and the expected transportation cost. Its aim is to minimize the expected total cost $H_{c}(s)$ :

$$
\begin{aligned}
H_{c}(s) & =\int_{0}^{\infty} \cdots \int_{0}^{\infty}\left\{\bar{h} \cdot\left[\sum_{i=1}^{n}\left(s_{i}-x_{i}\right)\right]^{+}+\bar{p} \cdot\left[\sum_{i=1}^{n}\left(x_{i}-s_{i}\right)\right]^{+}+\bar{t} \cdot \min \{S, D\}\right\} f(x) d x \\
& =\bar{H}_{c}\left(s_{0}\right)+\bar{t} \cdot \int_{0}^{\infty} \cdots \int_{0}^{\infty} \min \{S, D\} f(x) d x
\end{aligned}
$$

where

$$
\bar{H}_{c}\left(s_{0}\right)=\int_{0}^{\infty}\left(\bar{h} \cdot\left[s_{0}-x_{0}\right]^{+}+\bar{p} \cdot\left[x_{0}-s_{0}\right]^{+}\right) f_{0}\left(x_{0}\right) d x_{0}
$$

just the total expected cost of the Eppen's centralized system, and where $S=\sum_{i=1}^{n}\left[s_{i}-x_{i}\right]^{+}$ and $D=\sum_{i=1}^{n}\left[x_{i}-s_{i}\right]^{+}$, the total surplus and the total deficit in the whole system, respectively.

\section{The main results}

Main results of this paper consist of two parts stated as in theorem 1 and theorem 2:

Theorem 1. Let $\bar{s}^{*}$ be the optimal solution of problem (3.3) with the centralized expected cost $H_{c}\left(\vec{s}^{*}\right)$, and let $s^{*}$ be the optimal solution of problem (3.2) with the decentralized expected cost $H_{D}\left(s^{*}\right)$. Then

$$
\bar{t}<\bar{h}+\vec{p} \text { if and only if } H_{c}\left(\bar{s}^{*}\right)<H_{D}\left(s^{*}\right) .
$$

proof: First, note the properties of $[y]^{+}-[-y]^{+}=y$ and $\min \{x, y\}=y-[y-x]^{+}$for any $x$ and $y$. Then since

$$
S-D=\sum_{i=1}^{n}\left(s_{i}-x_{i}\right)=s_{0}-x_{0}
$$


we have

$$
\min \{S, D\}=D-[D-S]^{+}=\sum_{i=1}^{n}\left[x_{i}-s_{i}\right]^{+}-\left[x_{0}-s_{0}\right]^{+} .
$$

Furthermore, since

$$
\left[s_{0}-x_{0}\right]^{+}=\left[x_{0}-s_{0}\right]^{+}+s_{0}-x_{0}
$$

arranging Eq. (3.3) by substituting these into produces

$$
\begin{aligned}
H_{c}(s) & =(\bar{h}+\bar{p}-\bar{t}) \int_{0}^{\infty}\left[x_{0}-s_{0}\right]^{+} f_{0}\left(x_{0}\right) d x_{0}+\bar{h} \int_{0}^{\infty}\left(s_{0}-x_{0}\right) f_{0}\left(x_{0}\right) d x_{0} \\
& +\bar{t} \int_{0}^{\infty} \cdots \int_{0}^{\infty} \sum_{i=1}^{n}\left[x_{i}-s_{i}\right]^{+} f(x) d x
\end{aligned}
$$

Furthermore, (3.2) can be also arranged as

$$
H_{D}(s)=(\bar{h}+\bar{p}) \int_{0}^{\infty} \cdots \int_{0}^{\infty} \sum_{i=1}^{n}\left[x_{i}-s_{i}\right]^{+} f(x) d x+\bar{h} \int_{0}^{\infty}\left(s_{0}-x_{0}\right) f_{0}\left(x_{0}\right) d x_{0}
$$

From (4.4) and (4.5) we have

$$
\begin{aligned}
H_{D}(s)-H_{c}(s) & =(\bar{h}+\bar{p}-\bar{t})\left(\int_{0}^{\infty} \cdots \int_{0}^{\infty} \sum_{i=1}^{n}\left[x_{i}-s_{i}\right]^{+} f(x) d x-\int_{0}^{\infty}\left[x_{0}-s_{0}\right]^{+} f_{0}\left(x_{0}\right) d x_{0}\right) \\
& =(\bar{h}+\bar{p}-\bar{t})\left(\int_{0}^{\infty} \cdots \int_{0}^{\infty} \sum_{i=1}^{n}\left[x_{i}-s_{i}\right]^{+} f(x) d x-\int_{0}^{\infty} \cdots \int_{0}^{\infty}\left[\sum_{i=1}^{n}\left(x_{i}-s_{i}\right)\right]^{+} f(x) d x\right) \\
& =(\bar{h}+\bar{p}-\bar{t})\left(\int_{0}^{\infty} \cdots \int_{0}^{\infty}\left(\sum_{i=1}^{n}\left[x_{i}-s_{i}\right]^{+}-\left[\sum_{i=1}^{n}\left(x_{i}-s_{i}\right)\right]^{+}\right) f(x) d x\right.
\end{aligned}
$$

Note that $\sum_{i=1}^{n}\left[x_{i}-s_{i}\right]^{+}-\left[\sum_{i=1}^{n}\left(x_{i}-s_{i}\right)\right]^{+} \geq 0$ and that $\sum_{i=1}^{n}\left[x_{i}-s_{i}\right]^{+}-\left[\sum_{i=1}^{n}\left(x_{i}-s_{i}\right)\right]^{+}>0$ for $\left(x_{1}, x_{2}, \cdots, x_{n}\right) \in A^{c}$ where $A=\left\{\left(x_{1}, x_{2}, \cdots, x_{n}\right) \mid x_{i} \geq s_{i}\right.$ for all $i=1,2, \cdots, n$ or $x_{i} \leq s_{i}$ for all $i=1,2, \cdots, n$. $\}$.

Therefore,

$$
\int_{0}^{\infty} \cdots \int_{0}^{\infty}\left(\sum_{i=1}^{n}\left[x_{i}-s_{i}\right]^{+}-\left[\sum_{i=1}^{n}\left(x_{i}-s_{i}\right)\right]^{+}\right) f(x) d x>0
$$

From (4.6) and (4.7), we have

$$
\bar{t}<\bar{h}+\bar{p} \text { if and only if } H_{c}(s)<H_{D}(s) \text {, for all } s \text { and hence } H_{c}\left(\bar{s}^{*}\right)<H_{D}\left(s^{*}\right) \text {. }
$$

Theorem 2. Suppose that $p_{i j}$ 's, the correlation coefficients between $X_{i}$ and $X_{j}$, are all equal to 1 . Let $\bar{s}^{*}$ and $s^{*}$ be the optimal solution of problem (3.3) and problem (3.2), respectively. Then

$$
\begin{aligned}
& \text { (i) } \bar{s}^{*}=s^{*} \text {, and } \\
& \text { (ii) } H_{c}\left(\bar{s}^{*}\right)=H_{D}\left(s^{*}\right) \text {. }
\end{aligned}
$$

Proof: The assumption of $p_{i j}=1$ for all $i \neq j$, implies that there exist constants $b_{2}, b_{3}, \cdots$, $b_{n}$ and positive constants $a_{2}, a_{3} \cdots, a_{n}$, satisfying

$$
X_{i}=a_{i} X_{1}+b_{i}, i=2,3, \cdots, n,([2], \text { p. } 251) .
$$


For the decentralized system, we can set $s_{i}=a_{i} s_{1}+b_{i}, i=1,2, \cdots, n$, because the following transformation can be made for $H_{i}\left(s_{i}\right)$

$$
\begin{aligned}
H_{i}\left(s_{i}\right) & =\int_{0}^{\infty}\left(\bar{h} \cdot\left[s_{i}-a_{i} x_{1}-b_{i}\right]^{+}+\bar{p} \cdot\left[a_{i} x_{1}+b_{i}-s_{i}\right]^{+}\right) f_{1}\left(x_{1}\right) d x \\
& =a_{i} \int_{0}^{\infty}\left(\bar{h} \cdot\left[s_{1}-x_{1}\right]^{+}+\bar{p} \cdot\left[x_{1}-s_{1}\right]^{+}\right) f_{1}\left(x_{1}\right) d x_{1} \\
& =a_{i} H_{1}\left(s_{1}\right), s_{1}=\left(s_{i}-b_{i}\right) / a_{i}
\end{aligned}
$$

Since for any $i=2,3, \cdots, n, s_{i}-X_{i}=a_{i}\left(s_{1}-X_{1}\right) \geq 0$ if and only if $s_{1}-X_{1} \geq 0$, so it must be $S \equiv 0$ or $D \equiv 0$ and hence $\bar{t} \cdot \min \{S, D\}=0$.

Therefore (3.3) yield that

$$
H_{c}(s)=\bar{H}_{c}\left(s_{0}\right) \text {. }
$$

Let $a_{1}=1, b_{1}=0, a_{0}=\sum_{i=1}^{n} a_{i}, b_{0}=\sum_{i=1}^{n} b_{i}$ and let $s_{0}^{*}$ be the optimal solution of problem (3.4) with Eppen's centralized expected cost $\bar{H}_{c}\left(s_{0}^{*}\right)$. Then since

$$
X_{0}=\sum_{i=1}^{n} X_{i}=a_{0} X_{1}+b_{0}
$$

we can also set $s_{0}=a_{0} s_{1}+b_{0}$ in the same way as in (4.8).

Hence $s_{i}^{*}=a_{i} s_{1}^{*}+b_{i}$ for all $i=0,1, \cdots, n$.

This implies

$$
\begin{aligned}
s_{0}^{*}=a_{0} s_{1}^{*}+b_{0} & =\left(\sum_{i=1}^{n} a_{i}\right) s_{1}^{*}+\left(\sum_{i=1}^{n} b_{i}\right) \\
& =\sum_{i=1}^{n}\left(a_{i} s_{1}^{*}+b_{i}\right) \\
& =\sum_{i=1}^{n} s_{i}^{*}
\end{aligned}
$$

Combining (4.8) and (3.2), it leads to:

$$
\begin{aligned}
H_{D}\left(s^{*}\right) & =\sum_{i=1}^{n} H_{i}\left(s_{i}^{*}\right) \\
& =\sum_{i=1}^{n} a_{i} H_{1}\left(s_{1}^{*}\right) \\
& =a_{0} H_{1}\left(s_{1}^{*}\right) \\
& =\bar{H}_{c}\left(s_{0}^{*}\right)
\end{aligned}
$$

in which the last term is verified in the same way as in (4.8).

From (4.9) and (4.10), we have

Therefore,

$$
H_{D}(s)=H_{c}(s)
$$

$$
\begin{aligned}
H_{c}\left(\bar{s}^{*}\right) & =\min _{s} H_{c}(s) \\
& \geq \min _{s_{0}} \bar{H}_{c}\left(s_{0}\right) \\
& =\bar{H}_{c}\left(s_{0}^{*}\right) ; \text { by }(4.10) \\
& =H_{D}\left(s^{*}\right) ; \text { by }(4.11) \\
& =H_{c}\left(s^{*}\right)
\end{aligned}
$$


Accordingly, we have

$$
H_{c}\left(\bar{s}^{*}\right) \geq H_{D}\left(s^{*}\right)=H_{c}\left(s^{*}\right) \geq H_{c}\left(\bar{s}^{*}\right)
$$

implying

$$
H_{c}\left(\bar{s}^{*}\right)=H_{D}\left(s^{*}\right)=H_{c}\left(s^{*}\right)
$$

hence

$$
\bar{s}^{*}=s^{*}
$$

\section{Conclusions}

The measurement of the effects of centralization could be stated as follows:

(1) The effect of the centralized system depends on the value of $(\bar{h}+\bar{p})-\bar{t}$, if $(\bar{h}+\bar{p})-\bar{t}$ is larger, then it is more effective to adopt the centralized inventory policy.

(2) If $p_{i j}$, the coefficient of correlation of location's demands, is equal to 1 , the effect of the centralized system is completely unaffected by $\bar{t}$.

\section{Acknowledgements}

The authors wish to thank the referees for their helpful comments and suggestions.

\section{References}

[1] Eppen, G.D.: Effect of Centralization on Expected Costs in a Multi-location Newsboy Problem, Mgmt Sci.; Vol. 25 (1979), 498-501.

[2] Rohatgi, V.K.: Statistical Inference, Wiley, New York, (1984).

[3] Stulman, A.: Benefits of Centralized Stocking for the Multi-centre Newsboy Problem with First Come, First Served Allocation, Journal of the Operational Research Society, Vol. 38 (1987), 827-832.

Chin-Tsai Lin

Institute of Management Science

National Chiao-Tung University

and

Department of Mathematics

Tamkang University

Taiwan 